\title{
Solving reliability analysis problems in the polar space
}

\author{
Ghasem Ezzati*, Musa Mammadov, Siddhivinayak Kulkarni \\ Faculty of Science and Technology, Federation University, Mount Helen, Victoria, Australia \\ *Corresponding author E-mail: g.ezzati@federation.edu.au
}

Copyright (C)2014 Ezzati et. al. This is an open access article distributed under the Creative Commons Attribution License, which permits unrestricted use, distribution, and reproduction in any medium, provided the original work is properly cited.

\begin{abstract}
An optimization model that is widely used in engineering problems is Reliability-Based Design Optimization (RBDO). Input data of the RBDO is non-deterministic and constraints are probabilistic. The RBDO aims at minimizing cost ensuring that reliability is at least an accepted level. Reliability analysis is an important step in two-level RBDO approaches. Although many methods have been introduced to apply in reliability analysis loop of the RBDO, there are still many drawbacks in their efficiencies and stabilities. This paper proposes a novel method that converts the constrained reliability analysis problem to an unconstrained minimization problem in order to improve efficiency of the solution. Number of numerical experiments is conducted and then performance of the proposed method is compared with the existing reliability analysis methods.
\end{abstract}

Keywords: Polar space, Reliability analysis, Reliability-based design optimization

\section{Introduction}

Uncertainty plays an important role in system design. Reliability-based design optimization (RBDO) is an optimization model that takes into account uncertainties [10].

There are two loops in an RBDO problem which are reliability analysis and design optimization. Existing RBDO approaches with respect to manner of considering these loops are classified as mono-level, two-level and decoupled approaches [1].

Two nested loops have been considered in the two-level RBDO approaches. Inner loop carries out reliability analysis and outer-loop concerns about design optimization.

Reliability index approach (RIA) and performance measure approach (PMA) are two-loop RBDO approaches. Main difference between RIA and PMA is in their inner loops [1,9].

Inner loop of the RIA includes a first-order reliability analysis problem. Hasofer and Lind - Rackwitz and Fiessler (HL-RF) method is a commonly accepted method to solve this problem [8, 11, 12, 15, 16].

Reliability analysis problem of the PMA is a first-order inverse reliability analysis problem. Inner loop of the PMA is intended to solve this problem [6, 18].

Although it has been reported that the existing reliability analysis methods are stable and efficient to apply in inner loop of PMA [9, 20], a new method will be introduced in this paper to improve efficiency of the existing methods.

Moreover, general optimization algorithms, such as sequential linear programming (SLP) and sequential quadratic programming (SQP), can be used to solve reliability analysis problems of RIA and PMA [2, 3]. 
This paper has been designed as following. Next section introduces a general model of the reliability-based design optimization (RBDO). Then, various categories of RBDO approaches will be illustrated. After that, existing reliability analysis methods will be explained. In the following section, a new reliability analysis method will be introduced and then some numerical experiments will be solved. Lastly, a conclusion will be resulted based on the solved numerical experiments.

\section{A general model of Reliability-Based Design Optimization (RBDO)}

Reliability-based design optimization (RBDO) aims at searching for the best compromise between cost reduction and safety assurance based on the evaluation of probabilistic constraints. In other words, the RBDO not only provides a cost-effective manufacturing process, but also their requested confidence level [10].

Main features of the RBDO are non-deterministic input data and probabilistic constraints. These features make RBDO a complicated optimization model. It can be said that RBDO ensures a minimum total cost (a summation of initial cost (design and construction costs), failure cost and maintenance cost) without affecting target reliability level $[10,21]$.

In the RBDO process, random variables characterize physical quantities under uncertainties. In general, the statistical description of failure probability of a system in terms of random variables $(X)$ is determined as following [18]:

$$
P[G(X)<g]=F_{G}(g)=\iint \ldots \int_{G(X)<g} f_{X}(x) d X
$$

where $\mathrm{P}, \mathrm{F}$ and $\mathrm{f}$ are probability function, cumulative distribution function (CDF) and joint probability density function (JPDF), respectively. Also, $g$ is probabilistic performance measure and the random variable is bounded by its lower and upper bounds, $X^{L}$ and $X^{U}$, respectively.

Basic RBDO formulation consists of minimizing the cost function under probabilistic constraints. The random variable may be either independent deterministic variable (like $X$ ) or probability distribution parameter (like $\mu_{X}$ that is the expected value of $X$ ).

However, an RBDO model is generally written as below $[1,9,10]$ :

$$
\begin{array}{ll}
\text { Min } & \operatorname{Cost}(X) \\
\text { s.t. } & P_{f_{j}} \leq \bar{P}_{f_{j}} \quad j=1,2, \ldots, n p f \\
& X^{L} \leq X \leq X^{U}
\end{array}
$$

where the cost function can be any function of the random variable, $P_{f_{j}}$ is the failure probability of $j-t h$ performance function and $\bar{P}_{f_{j}}$ is a predetermined acceptable failure probability limit of $j$ - th performance function.

$\bar{P}_{f}$ is often set on the basis of engineering knowledge and experience with respect to previous designs. Also, upper and lower bounds of the random variables are typically known as deterministic constraints [10].

The probabilistic constraint defines feasible region by restricting the probability of violating limit state $(P[(G(X))<$ $0])$ to an admissible failure probability $\left(\bar{P}_{f}=\Phi\left(-\beta_{t}\right)\right.$ where $\Phi$ is standard normal CDF and $\beta_{t}$ is target reliability index) [18].

\section{Main approaches of RBDO}

There are three groups of approaches that perform these levels using various methods. Different RBDO approaches will be illustrated in the following subsections shortly.

\subsection{Mono-Level approaches}

The first method to consider a reliability analysis problem in an RBDO problem is to avoid the reliability analysis loop. This manner is used in mono-level RBDO approaches. In fact, there is just one loop in this category of RBDO approaches [1].

In this case, the probabilistic constraints are replaced by optimality conditions. This replacement should be done such that the RBDO problem can be reformulated in a mono-level optimization problem. 
Karush-Kahn-Tucker (KKT) optimality condition is the first approach of this category. The KKT optimality conditions are applied in the RBDO problem and computational cost is saved. However, reduction of total cost is not significant $[1,14]$.

Single-loop single-vector (SLSV) is another mono-level RBDO approach. This approach approximates the performance function of RBDO problem. In this approach, optimum point is calculated using target reliability index and limit-state derivatives [4].

Approximate moment approach (AMA) is a probabilistic mono-level RBDO approach. This approach is based on robust design optimization [6]. Although there is no need to reliability analysis problems in the AMA, a large amount of computational effort is still required in this approach. This happens because second-order sensitivity analysis is required [21].

\subsection{Two-Level approaches}

Two nested loops are involved in two-level RBDO approaches in order to solve reliability analysis and design optimization problems. Reliability index approach (RIA) and performance measure approach (PMA) are two-level RBDO approaches.

RIA includes a first-order reliability analysis problem in its inner loop (reliability analysis loop) that must be solved in the standard normalized random space ( $U$-space) [15]. This problem searches for the nearest point on the limit-state function to the origin of the $U$-space [8].

In the other two-level RBDO approach (PMA) a first-order inverse reliability analysis problem is considered. PMA was introduced based on this fact that minimizing a complicated cost function subject to a simple constraint is easier than minimizing a simple cost function subject to a complicated constraint $[1,18]$.

The performance function of a reliability analysis problem in PMA is converted to a performance measure. In fact, the performance function should be minimized on the target reliability surface in the PMA.

Furthermore, sampling-based method, such as monte carlo simulation (MCS), are used to solve reliability analysis problems of two-level RBDO approaches. In this case, size of MCS, realization of random variables and accepted failure probability are used to approximate the probabilistic constraint [19, 22].

\subsection{Decoupled approaches}

In the third category of RBDO approaches, reliability analysis and design optimization problems are decoupled. In these approaches, an RBDO problem is transformed to a sequence of deterministic design optimization problems [1]. Thus, the design optimization loop does not carry the reliability analysis problem.

Sequential optimization and reliability assessment (SORA) is one of the commonly accepted decoupled RBDO approaches. Design point is improved from cycle to cycle in this method [7].

The SORA method modifies efficiency of the probabilistic optimization by a serial-loop strategy with a cycle of reliability analysis and deterministic design optimization.

Sequential approximate programming (SAP) is another decoupled RBDO approach. The RBDO problem is reformulated as a sub-programming problem in this approach. The first-order Taylor series at the current design point is used instead of the probabilistic constraint [5].

\section{Reliability analysis problems}

Safety is an important property of all engineering systems. Due to this importance, many efforts have been done to improve systems' safety.

Reliability analysis problems intend to guarantee a level of assurance for a system. However, it is impossible to reach an absolute safety for an engineered system, because there are many sources of uncertainties for such a system [3].

In this paper, we talk about reliability analysis problems of performance measure approach (PMA). In this regard, the main task of a reliability analysis problem is to evaluate probabilistic constraint of an RBDO problem when the PMA is applied.

In general, reliability analysis problems are often solved in the standard normal random space ( $U$-space). Thus, it's required to transform a reliability analysis problem from the original random space ( $X$-space) to the $U$-space.

First-order reliability method (FORM) is a widely accepted tool to transform a problem from the $X$-space to the $U$-space; i.e. $T: X \rightarrow U[18]$. 
This transformation is constructed regarding probability distribution function of random variables. For example, if the random variables were normally distributed, then $T$ is defined as following:

$$
u=\frac{x-\mu}{\sigma}
$$

where $x$ and $u$ are original and standard normalized random variables, respectively. Also, $\mu$ and $\sigma$ are statistical parameters (mean value and standard deviation of $x$, respectively).

Stability of a reliability analysis method has a considerable role in two-level optimization problems (such as RBDO problems). Because if it diverges in the inner loop of an RBDO problem, the total process is stopped and cannot find answer of main problem.

Furthermore, since the reliability analysis problem is often not a convex problem, it's not possible to find the global optimum solution.

Two different reliability analysis problems are used in inner loop of two-level RBDO approaches.

A first-order reliability analysis problem, which is applied in the RIA, tries to find optimum point on the standard normalized failure surface $\left(G_{U}(U)=0\right)$. This point should have the minimum distance from the origin of the $U$-space. This distance is considered as safety reliability index.

The first-order reliability analysis problem is as below [1]:

$$
\begin{aligned}
& \text { Min }\|u\| \\
& \text { s.t. } G_{U}(u)=0
\end{aligned}
$$

where variable $u$ is the transformed random variable in the $U$-space. The optimum point of this problem is called most probable failure point (MPFP).

Any general optimization algorithm can be used to solve this problem. Also, Hasofer and Lind - Rackwitz and Fiessler (HL-RF) method is accepted to apply in the inner loop of RIA [18].

The HL-RF method was proposed firstly for second-moment reliability analysis problems and then improved to include distributed information $[11,16]$.

The HL-RF method can also be applied to solve optimization problems of structural reliability [13].

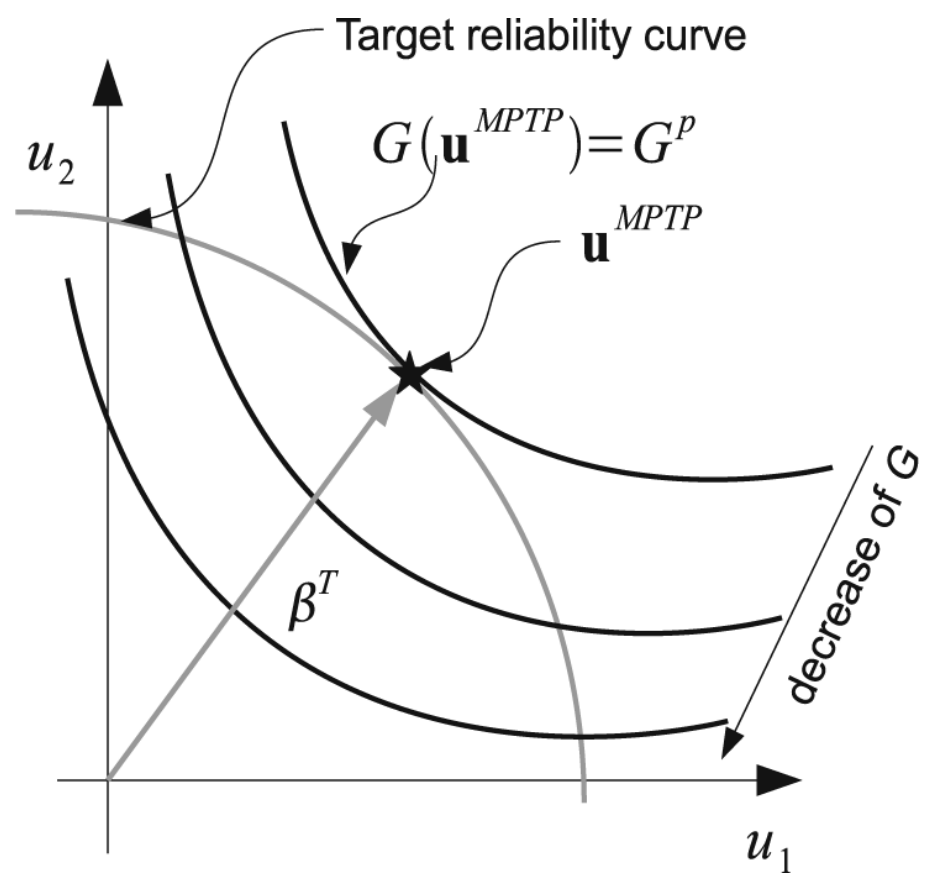

Figure 1: First-order inverse reliability analysis problem applied in the PMA; optimum point of this problem is called minimum performance target point (MPTP) that has the minimum amount of standard normalized performance function on the target reliability surface.

Moreover, inner loop of the performance measure approach (PMA) includes a first-order inverse reliability analysis problem. 
This problem minimizes $G_{U}(U)$ (standard normalized performance function) on the target reliability surface (i.e. $\|U\|=\beta_{t}$ ). Figure 1 displays reliability analysis problem of the PMA.

Obtained answer of this problem is used to find probabilistic performance measure $\left(g^{*}\right)$ such that the probabilistic constraint of the PMA is constructed.

The probabilistic constraint of an RBDO problem, when the PMA is applied, is supposed as $g^{*} \geq 0$ [18].

Reliability analysis problem of the PMA is defined as below [1, 9]:

$$
\begin{aligned}
& \text { Min } G_{U}(u) \\
& \text { s.t. }\|u\|=\beta_{t}
\end{aligned}
$$

where variable $u$ is the standard normalized random variable and $\beta_{t}$ is target reliability index.

We will introduce a method in the next section that can be applied to solve reliability analysis problem of the PMA. Performance of this method will be compared with the existing methods.

The existing reliability analysis methods are explained in the following subsections.

\subsection{Hybrid mean value method}

The steepest descent direction is firstly applied to solve reliability analysis problem of the PMA. In this regard, hybrid mean value (HMV) method was introduced to solve this problem [20].

The HMV method uses a function type criterion to determine type of performance function. This criterion uses three consecutive directions as below:

$$
\zeta^{(k+1)}=\left(n^{(k+1)}-n^{(k)}\right) \cdot\left(n^{(k)}-n^{(k-1)}\right)
$$

where $\zeta^{(k+1)}$ describes type of the performance function at the $(k+1)^{t h}$ iteration. Also, $n^{(k)}$ is the steepest descent direction of the performance function at the $k^{t h} \operatorname{design}$ point $\left(u_{H M V}^{(k)}\right)$.

The HMV method can be summarized as following [20]:

1. Set the iteration counter $k=0$. Select the convergence parameter $\varepsilon$. Compute:

$$
n\left(u_{H M V}^{(0)}\right)=-\frac{\nabla_{U} G\left(u_{H M V}^{(0)}\right)}{\left\|\nabla_{U} G\left(u_{H M V}^{(0)}\right)\right\|}
$$

where $u_{H M V}^{(0)}=0$.

2. If $k<3$ or $\zeta^{(k+1)}$ is positive, then:

$$
u_{H M V}^{(k+1)}=\beta_{t} \cdot n\left(u_{H M V}^{(k)}\right)
$$

If $k \geq 3$ and $\zeta^{(k+1)}$ is zero or negative, then:

$$
u_{H M V}^{(k+1)}=\beta_{t} \cdot \frac{n\left(u_{H M V}^{(k)}\right)+n\left(u_{H M V}^{(k-1)}\right)+n\left(u_{H M V}^{(k-2)}\right)}{\left\|n\left(u_{H M V}^{(k)}\right)+n\left(u_{H M V}^{(k-1)}\right)+n\left(u_{H M V}^{(k-2)}\right)\right\|}
$$

Note that: $n\left(u_{H M V}^{(k)}\right)=-\frac{\nabla_{U} G\left(u_{H M V}^{(k)}\right)}{\left\|\nabla_{U} G\left(u_{H M V}^{(k)}\right)\right\|}$

3. Calculate $G_{U}\left(u_{H M V}^{(k+1)}\right)$. Check the convergence criterion $\left(\left|G\left(u^{(i)}\right)-G\left(u^{(i-1)}\right)\right|<\varepsilon\right)$.

If it's held, then stop; otherwise, go to the next step.

4. Check $\zeta^{(k+1)}$ and set $k=k+1$. Then return to the second step.

It is found that the HMV method is stable and efficient to evaluate performance functions [1, 20]. 


\subsection{Conjugate gradient analysis method}

The conjugate gradient direction has been applied to introduce another reliability analysis method. In this regard, conjugate gradient analysis (CGA) method is introduced as a reliability analysis method [9].

In this method, the steepest descent direction, applied in the HMV method, was replaced by the conjugate gradient direction in order to improve stability and efficiency of the reliability analysis procedure.

The conjugate gradient analysis method can be summarized as below [9]:

1. Set the iteration counter $i=0$.

Select the convergence parameters $\varepsilon_{1}, \varepsilon_{2}$ and $\varepsilon_{3}$.

Suppose:

$$
d_{0}=0, u^{(0)}=0_{n * 1}, w^{(0)}=0_{n * 1}
$$

where $n$ is the number of random variables in the problem.

2. Calculate $G_{U}\left(u^{(0)}\right)$.

3. Calculate $w^{(i+1)}=-\nabla G_{U}\left(u^{(i)}\right)+d_{i} \cdot w^{(i)}$

4. Calculate $u^{(i+1)}=\beta_{t} \frac{w^{(i+1)}}{\left\|w^{(i+1)}\right\|}$

5. Calculate $G_{U}\left(u^{(i+1)}\right)$.

6. Calculate $d_{i+1}=\frac{\left\|\nabla G_{U}\left(u^{(i+1)}\right)\right\|^{2}}{\left\|\nabla G_{U}\left(u^{(i)}\right)\right\|^{2}}$.

7. Check the following stopping criteria:

$$
\left\|u^{(i)}-u^{(i-1)}\right\|<\varepsilon_{1},\left\|\nabla G_{U}\left(u^{(i-1)}\right)\right\|<\varepsilon_{2} \text { and }\left|G_{U}\left(u^{(i)}\right)-G_{U}\left(u^{(i-1)}\right)\right|<\varepsilon_{3}
$$

8. If at least one stopping criterion is held, then stop. Otherwise, set $i=i+1$ and go to step 3 .

It has been reported in the literature that the CGA method is more stable and efficient than the HMV method to solve reliability analysis problems [9].

\section{Unconstrained polar reliability analysis method}

Reliability analysis problem of the PMA is often transformed from the original random space ( $X$-space) to the standard normal random space ( $U$-space).

It means that the original performance function $\left(G_{X}(X)\right)$ in the inner loop of an RBDO problem must be standard normalized. In other words, it should be transformed to $G_{U}(U)$.

RIA and PMA include different reliability analysis problems. It has been reported that PMA results better stability and efficiency than RIA [1, 9, 18]. Therefore, we will compare our new method with the HMV and CGA methods.

In this paper, we will transform the standard normalized performance function from the $U$-space to the polar co-ordinate system.

It has to be noted that direct transformation from the original random space to the polar co-ordinate system is difficult and sometimes impossible. In this case, if the statistical parameters were different, specially when the standard deviations of the random variables are not the same, a square root function will be involved in the problem that makes the problem complicated.

When this transformation is done in the $U$-space, the equality constraint (i.e. $\|U\|=\beta_{t}$ ) can be simply converted to $\rho=\beta_{t}$ where $\rho$ is the radius of a circle or sphere in the two or three dimensional spaces, respectively. $\rho$, which is a fixed number, is used in the convertors from the $U$-space to the polar co-ordinate system. 
The basic idea of this conversion is to reduce the number of variables in the reliability analysis problem. The equality constraint of the reliability analysis problem shows a circular / spherical constraint with a fixed radius in the two / three dimensional spaces, respectively. Thus, it can be concluded that the number of variables is reduced from $n$ to $n-1$.

Moreover, it must be considered that the reliability analysis problem, which is a constrained minimization problem in the $U$-space, will be changed to an unconstrained minimization problem by converting to the polar co-ordinate system.

It happens because there is only one constraint in the original reliability analysis problem. This constraint can be generally seen as a fixed number as radius in the new space.

Thus, it's predictable that evaluating functions in the polar co-ordinate system has less difficulties than evaluating them in the standard normal random space.

In this new method, the performance function in the $U$-space should be re-written in the polar co-ordinate system. For this purpose, the random variables must be converted to a product of trigonometric functions.

Since the constrained reliability analysis problem is converted to an unconstrained reliability analysis problem in the polar co-ordinate system, this new method will be called "Unconstrained Polar Reliability Analysis (UPRA) Method".

Required relationships to convert functions from the $U$-space to the polar co-ordinate system are as below:

Two dimensional space:

$U_{1}=r \cdot \cos (\theta)$

$U_{2}=r . \sin (\theta)$

where $r$ is a constant (radius of the circle) and $\theta$ is the polar angle so that:

$$
U_{1}^{2}+U_{2}^{2}=r^{2}
$$

\section{Three dimensional space:}

$U_{1}=\rho \cdot \sin (\phi) \cdot \cos (\theta)$

$U_{2}=\rho \cdot \sin (\phi) \cdot \sin (\theta)$

$U_{3}=\rho \cdot \cos (\phi)$

where $\rho$ is a constant (radius of the sphere), $\theta$ is the polar angle and $\phi$ is the azimuthal angle so that:

$$
U_{1}^{2}+U_{2}^{2}+U_{3}^{2}=\rho^{2}
$$

These transformers can be expanded for $n$-dimensional problems. Therefore, we have:

\section{$n$ dimensional space:}

$U_{1}=\rho \cdot \sin \left(\theta_{1}\right) \cdot \sin \left(\theta_{2}\right) \cdot \sin \left(\theta_{3}\right) \ldots \sin \left(\theta_{n-2}\right) \cdot \cos \left(\theta_{n-1}\right)$

$U_{2}=\rho \cdot \sin \left(\theta_{1}\right) \cdot \sin \left(\theta_{2}\right) \cdot \sin \left(\theta_{3}\right) \ldots \sin \left(\theta_{n-2}\right) \cdot \sin \left(\theta_{n-1}\right)$

$U_{3}=\rho \cdot \sin \left(\theta_{1}\right) \cdot \sin \left(\theta_{2}\right) \cdot \sin \left(\theta_{3}\right) \ldots \cos \left(\theta_{n-2}\right)$

$U_{4}=\rho \cdot \sin \left(\theta_{1}\right) \cdot \sin \left(\theta_{2}\right) \ldots \cos \left(\theta_{n-3}\right)$

and so on, until:

$U_{n-1}=\rho \cdot \sin \left(\theta_{1}\right) \cdot \cos \left(\theta_{2}\right)$

$U_{n}=\rho \cdot \cos \left(\theta_{1}\right)$

where $\rho$ is a constant. Also, we have:

$$
U_{1}^{2}+U_{2}^{2}+\ldots+U_{n}^{2}=\rho^{2}
$$

When the reliability analysis problem is changed to an unconstrained optimization problem, it can be solved using any general unconstrained optimization algorithm.

In this paper, the obtained function in the polar co-ordinate system will be minimized using the steepest descent method.

If a reliability analysis problem has $n$ random variables (after transforming problem to the polar space), only $k$ first components of the gradient vector are non-zero in iteration $k$.

It happens because of the nature of transformations from the $U$-space to the polar space that involves trigonometric functions. Thus, in iteration $k$, just $k$ first components of design point are changed.

Therefore, it can be concluded that in a $n$-dimensional problem, which is changed to a $(n-1)$-dimensional problem in the polar space, the first iteration in which all components of the gradient vector are non-zero and so all components of design point are updated is $(n-1)^{t h}$ iteration.

Moreover, to integrate the UPRA method with an RBDO problem it must be mentioned that an RBDO problem, as a two-level problem, deals with reliability analysis and design optimization. 
In general, we start by an initial design point. To update this point, it's required to solve a reliability analysis problem.

After solving this problem, obtained information must be used to calculate a new design point (by updating current design point in the design optimization level).

In the following section, number of numerical experiments will be solved in order to make a comparison between performances of the HMV and CGA methods (as the existing reliability analysis methods) and the UPRA method introduced in this paper.

\section{Numerical experiments}

In this section, some numerical problems are presented and solved. Different reliability analysis methods (existing HMV and CGA methods and new UPRA method) have been applied to solve these experiments.

There are three subsections here.

The first subsection includes five two- and three dimensional problems. Initial design points are fixed in this subsection.

The same problems will be solved in the second subsection, but in this subsection their initial design points are randomly generated by MATLAB software. 50 initial design points are generated and used for each problem.

In the third subsection, a particular function will be introduced and minimized using the mentioned methods. Dimension of this function will be changed from 2 to 10.

Number of required iterations for convergence, obtained values of performance functions and elapsed CPU times will be used to compare performances of different methods.

Furthermore, it must be noted that in the following problems, the accepted tolerances of the stopping criteria in all cases are considered equal $10^{-5}$. Also, all CPU times are shown in seconds in this paper.

All algorithms have been implemented in MATLAB. The associated codes are made available upon request.

\section{1. $\quad$ Fixed initial design point}

Problem 1

Consider the following performance function.

$$
G(X)=\frac{\left(x_{1}+x_{2}-5\right)^{2}}{30}+\frac{\left(x_{1}-x_{2}-12\right)^{2}}{120}-1
$$

where $X_{1} \sim N(1,0.6)$ and $X_{2} \sim N(2.3,0.8)$. Also, the target reliability index $\left(\beta_{t}\right)$ equals 2 .

The given performance function in this experiment is minimized using three methods. Table 1 displays numerical details obtained by these methods.

Table 1: Minimizing $G(X)$ using different reliability analysis methods to find MPTP

\begin{tabular}{lccc}
\hline & HMV & CGA & UPRA \\
\hline Iterations & 14 & 9 & 3 \\
$X_{1}$ & 2.1091 & 2.1094 & 2.0770 \\
$X_{2}$ & 1.6891 & 1.6902 & 1.5944 \\
$G(X)$ & 0.1656 & 0.1656 & 0.1643 \\
Elapsed Time & 0.026 & 0.016 & 0.021 \\
\hline
\end{tabular}

It can be seen in the Table 1 that all methods are convergent in this problem.

Various methods obtain various optimum points. However, required iterations of the new UPRA method is less than the other methods.

Further, the UPRA method obtains a value of performance function better than the existing HMV and CGA methods. 
Problem 2

A performance function is given as below:

$$
G(X)=3 e^{-X_{1}+X_{2}}+2 X_{1}^{2}-4 X_{2}
$$

where $X_{1}$ and $X_{2}$ are normally distributed. Their statistical parameters are $(4,0.6)$ and $(2,0.4)$, respectively. Also, target reliability index equals 3 .

All methods are applied to minimize the performance function given in this problem.

Detailed results obtained using these methods are displayed in Table 2.

Table 2: Minimizing $G(X)$ using different reliability analysis methods to find MPTP

\begin{tabular}{lccc}
\hline & HMV & CGA & UPRA \\
\hline Iterations & 14 & 4 & 3 \\
$X_{1}$ & 2.2275 & 2.2275 & 2.2150 \\
$X_{2}$ & 2.2090 & 2.2090 & 2.1546 \\
$G(X)$ & 4.0326 & 4.0326 & 4.0182 \\
Elapsed Time & 0.022 & 0.013 & 0.018 \\
\hline
\end{tabular}

In this problem, again all methods are convergent. Both existing methods find the same optimum points, but the new UPRA method reaches another point.

It can be resulted from the table that the performance function value obtained by the UPRA method is better (less) than value found by the existing HMV and CGA methods.

Problem 3

Consider the following performance function.

$$
G(X)=0.3 X_{1}^{2} X_{2}-X_{2}+0.8 X_{1}+1
$$

where $X_{1} \sim N(0,0.55)$ and $X_{2} \sim N(6,0.55)$. Also, the target reliability index is supposed 2 .

Table 3 shows details of numerical results obtained using different methods.

Table 3: Minimizing $G(X)$ using different reliability analysis methods to find MPTP - The HMV method diverges in this problem

\begin{tabular}{lccc}
\hline & HMV & CGA & UPRA \\
\hline Iterations & 10000 & 9 & 4 \\
$X_{1}$ & - & -0.1549 & -0.1549 \\
$X_{2}$ & - & 7.0890 & 7.0890 \\
$G(X)$ & Diverged & -6.1619 & -6.1619 \\
Elapsed Time & 5.237 & 0.022 & 0.029 \\
\hline
\end{tabular}

When the HMV method is applied to solve this problem, an oscillating behavior starts from $31^{\text {st }}$ iteration. Thus, this method cannot find the optimum point and diverges. In this case, this algorithm is stopped after 10,000 iterations.

But the other methods are stable and find the same optimum point. Also, their obtained performance function values are the same.

Although the elapsed time of the UPRA method is a little more than the CGA method, the number of required iterations of the UPRA method is less than the CGA method.

Problem 4

A performance function is given as below:

$$
G(X)=2 X_{1}^{3}+3 X_{2} e^{X_{3}}
$$


where initial design point is $[1,4,-2]$ and the standard deviations are $[0.3,0.4,0.8]$. Also, the target reliability index is supposed 3.

Table 4 displays results obtained using all methods.

Table 4: Minimizing $G(X)$ using different reliability analysis methods to find MPTP

\begin{tabular}{lccc}
\hline & HMV & CGA & UPRA \\
\hline Iterations & 126 & 10 & 4 \\
$X_{1}$ & 0.2889 & 0.2884 & 0.3632 \\
$X_{2}$ & 3.8134 & 3.8147 & 3.8919 \\
$X_{3}$ & -3.4232 & -3.4217 & -3.6821 \\
$G(X)$ & 0.4213 & 0.4217 & 0.3897 \\
Elapsed Time & 0.049 & 0.017 & 0.027 \\
\hline
\end{tabular}

In this problem, all methods are stable, but they find different MPTPs. The UPRA method has the minimum number of required iterations for convergence.

It can be seen in the table that although the CGA method has the shortest elapsed time, its performance function value is the worst amount among all methods.

Again in this experiment, the best value of performance function is calculated by the new UPRA method.

Problem 5

Consider the following performance function.

$$
G(X)=\frac{e^{-X_{1}}+3 X_{2}^{3}-4 X_{3}^{2}}{2 X_{2} X_{3}^{2}+5 X_{1} X_{2} X_{3}}
$$

where $X_{1} \sim N(3,0.5), X_{2} \sim N(-2.3,0.3)$ and $X_{3} \sim N(1,0.8)$. Also, the target reliability index is supposed 3 .

The performance function given in this problem is minimized using all methods. Table 5 is used to show details of obtained answers.

Table 5: Minimizing $G(X)$ using different reliability analysis methods to find MPTP - The HMV method diverges in this problem

\begin{tabular}{lccc}
\hline & HMV & CGA & UPRA \\
\hline Iterations & 10000 & 36 & 109 \\
$X_{1}$ & - & 3.3630 & 4.1243 \\
$X_{2}$ & - & -1.4423 & -1.8853 \\
$X_{3}$ & - & 0.5622 & 2.1408 \\
$G(X)$ & Diverged & 0.7033 & 0.3822 \\
Elapsed Time & 5.419 & 0.021 & 0.118 \\
\hline
\end{tabular}

As can be seen in this table, the HMV method diverges in this problem and cannot find the optimum point (MPTP) even after 10,000 iterations. Thus, this algorithm is stopped after 10,000 iterations.

But the other methods are stable and find different optimum points.

It can be found from the table that although the number of iterations and also the elapsed time of the CGA method are less than the UPRA method, there is a significant difference between performance function values obtained $b$ the CGA and UPRA methods.

Value of performance function obtained by the UPRA method is much more better than the value found by the CGA method.

All above problems will be solved in the next subsection again using 50 different initial design points that are randomly generated by MATLAB. 


\subsection{Randomly generated initial design points}

The problems solved in the previous subsection are solved in this subsection again using 50 different initial design points.

The design points are randomly generated by the MATLAB software. Answers obtained using the HMV, CGA and UPRA methods are shown it Table 6.

Each table has three columns. The first column shows the number of cases in which the relevant method is convergent.

Also, the second and third columns represent averages of required iterations and obtained values of performance functions.

Table 6: Reliability analysis problem by randomly generated initial design points - The best performance function values are shown in bold.

\begin{tabular}{cccccccccc}
\hline & \multicolumn{3}{c}{ Convergence } & \multicolumn{3}{c}{ Iterations } & \multicolumn{3}{c}{ Function Value } \\
Problem & HMV & CGA & UPRA & HMV & CGA & UPRA & HMV & CGA & UPRA \\
\hline 1 & 50 & 50 & 50 & 10 & 9 & 2 & 1.2745 & 0.9253 & $\mathbf{0 . 6 9 0 2}$ \\
2 & 0 & 50 & 50 & 10000 & 16.4 & 2.9 & - & 11.3112 & $\mathbf{4 . 6 6 5 7}$ \\
3 & 50 & 50 & 50 & 9 & 51 & 3.1 & -2.0784 & -0.8461 & $\mathbf{- 2 . 3 8 3 6}$ \\
4 & 50 & 50 & 50 & 8 & 55 & 6.7 & -45.3117 & -53.2223 & $\mathbf{- 5 5 . 6 4 8 6}$ \\
5 & 0 & 50 & 50 & 10000 & 12.7 & 7.6 & - & 0.4294 & $\mathbf{0 . 3 8 5 3}$ \\
\hline
\end{tabular}

Based on the data displayed in the tables, we can conclude that the CGA and UPRA methods are stable in all problems, while the HMV method is sometimes divergent.

Furthermore, it can be resulted by comparing the averages of required iterations that the UPRA method requires the minimum number of iterations, generally.

Moreover, the averages of performance function values obtained using the UPRA method is much more better (less) than the performance function values obtained by other methods.

Thus, it can be concluded that the UPRA method is more stable and efficient than the existing HMV and CGA methods to minimize these performance functions.

\subsection{High dimensional problem}

The following performance function is given. Suppose that the number of variables $(n)$ can change between 2 and 10 .

$$
G(X)=\frac{\sum_{i=1}^{n} e^{-x_{i}^{i}}}{\prod_{i=1}^{n} x_{i}^{i+1}}
$$

where $\sigma=0.3$ and $\beta_{t}=3$. Also, the initial design point is $X^{(0)}=(2,2, \ldots, 2)$.

Table 7: Minimizing performance function $(G(X))$ using the HMV, CGA and UPRA Methods - The best performance function values are shown in bold.

\begin{tabular}{ccccccc}
\hline & & $G(X)$ & & Time \\
Problem & HMV & CGA & UPRA & HMV & CGA & UPRA \\
\hline 2 & 0.1700 & $\mathbf{0 . 0 2 1 2}$ & $\mathbf{0 . 0 2 1 2}$ & 0.022 & 0.018 & 0.049 \\
3 & 0.1310 & 0.0124 & $\mathbf{0 . 0 1 2 0}$ & 0.022 & 0.019 & 0.053 \\
4 & 0.0815 & 0.0068 & $\mathbf{0 . 0 0 6 6}$ & 0.024 & 0.020 & 0.059 \\
5 & 0.0487 & 0.0036 & $\mathbf{0 . 0 0 3 5}$ & 0.026 & 0.020 & 0.085 \\
6 & 0.0278 & 0.0019 & $\mathbf{0 . 0 0 1 8}$ & 0.037 & 0.022 & 0.112 \\
7 & 0.0155 & 0.0012 & $\mathbf{0 . 0 0 1 0}$ & 0.027 & 0.025 & 0.148 \\
8 & 0.0085 & $\mathbf{0 . 0 0 0 5}$ & $\mathbf{0 . 0 0 0 5}$ & 0.030 & 0.026 & 0.159 \\
9 & 0.0046 & $\mathbf{0 . 0 0 0 3}$ & $\mathbf{0 . 0 0 0 3}$ & 0.032 & 0.029 & 0.167 \\
10 & 0.0024 & $\mathbf{0 . 0 0 0 1}$ & $\mathbf{0 . 0 0 0 1}$ & 0.034 & 0.032 & 0.174 \\
\hline
\end{tabular}


This performance function is minimized using all methods for different number of variables. Table 7 shows obtained numerical results using the HMV, CGA and UPRA methods, respectively, for different conditions.

It can be found from the table that all methods are stable in all cases.

Although the number of required iterations are not displayed in the table (because of the space limitations), the new UPRA method needs the least number of iterations for convergence.

Further, the performance function values obtained using the new UPRA method are better than the values resulted from the other methods.

On the other hand, the elapsed time of the UPRA method is longer than the other methods. This has happened because of the transformations involved in the MATLAB code. In all cases, so many transformations are required in this experiment in order to calculate gradient vector and performance function value in the UPRA method.

\section{Conclusion}

A new reliability analysis method is introduced in this paper. This method is applied in inner loop of the performance measure approach (PMA). Stability and efficiency of this method is compared with the existing HMV and CGA methods.

Based on the solved numerical experiments, it can be concluded that the new UPRA method is always stable and convergent, while the HMV method is divergent in some cases.

In all cases, the UPRA method finds the best amounts of performance function. Thus, it can be concluded that the UPRA method is the most efficient reliability analysis method.

\section{Acknowledgements}

This research is partially supported by National ICT Australia (NICTA).

\section{References}

[1] Aoues, Y. and Chateauneuf, A., "Benchmark Study of Numerical Methods for Reliability-Based Design Optimization", Structural and Multidisciplinary Optimization, Vol.41, (2010), pp.247-294.

[2] Arora, J. S., Introduction to Optimum Design, Waltham, Academic Press, (2012).

[3] Ayyub, B. M. and McCuen, R. H., Probability, Statistics and Reliability for Engineers and Scientists, Boca Raton, CRC Press, (2011).

[4] Chen, D. and Hasselman, T. K. and Neill, F. J., "Reliability-Based Structural Design Optimization for Practical Application", 38th AIAA/ASME/ASCE/AHA/ASC Structures, Structural Dynamics and Material Conference, (1997).

[5] Cheng, G. and Xu, L. and Jiang, L., "A Sequential Approximate Programming Strategy for Reliability-Based Structural Optimization", Computer Structures, Vol.84, No. 21 (2006), pp.1353-1367.

[6] Choi, K. K. and Youn, B. D., "On Probabilistic Approaches for Reliability-Based Design Optimization", 9th AIAA/NASA/USA/ISSMO Symposium on Multidisciplinary Analysis and Optimization, (2002).

[7] Du, X. and Chen, W., "Sequential Optimization and Reliability Assessment Method for Efficient Probabilistic Design", Journal of Mechanical Design, Vol.126 (2004), pp.225-233.

[8] Enevoldsen, I. and Sorensen, J. D., "Reliability-Based Optimization in Structural Engineering", Structural Safety, Vol.15, No. 3 (1994), pp.169-196.

[9] Ezzati, G. and Mammadov, M. and Kulkarni, S., "A New Reliability Analysis Method based on the Conjugate Gradient Direction", Structural and Multidisciplinary Optimization, DOI: 10.1007/s00158-014-1113-z, (2014).

[10] Frangopol, D. M. and Tsompanakis, Y. and Lagaros, N. D. and Papadrakakis, M., Structural Design Optimization Considering Uncertainties, Taylor and Francis, London, (2008).

[11] Hasofer, A. M. and Lind, N. C., "Exact and Invariant Second-Moment Code Format", Journal of Engineering Mechanics, Vol. 100, No. 1 (1974), pp. 111-121. 
[12] Liu, P. L. and Kiureghian, A. D., "Optimization Algorithms for Structural Reliability", Structural Safety, Vol.9 (1991), pp.161-177.

[13] Madsen, H. O. and Krenk, S. and Lind, N. C., Methods of Structural Safety, Prentice-Hall, Englewood Cliffs, (1986).

[14] Madsen, H. O. and Hansen, F., "A Comparison of Some Algorithms of reliability-based design optimization", 4th IFIP WG 7.5 Conference, (1992), pp. 443-451.

[15] Nikoladis, E. and Burdisso, R., "Reliability-Based Optimization: a Safety Index Approach", Computer Structures, Vol.28, No. 6 (1988), pp.781-788.

[16] Rackwitz, R. and Fiessler, B., "Structural Reliability under Combined Load Sequences", Computer Structures, Vol.9 (1978), pp.489-474.

[17] Tu, J. and Choi, K. K., "A Performance Measure Approach in Reliability-Based Structural Optimization", Master's Thesis, University of Iowa, (1997).

[18] Tu, J. and Choi, K. K. and Park, Y. H., "A New Study on Reliability-Based Design Optimization", Journal of Mechanical Design, Vol.121, No. 4 (1999), pp.557-564.

[19] Wang, H. and Gong, Z. and Huang, H. Z. and Zhang, Z. and Lv, Z., "System Reliability Based Design Optimization with Monte Carlo Simulation", IEEE Journal, Vol.12 (2012), pp.1143-1147.

[20] Youn, B. D. and Choi, K. K. and Park, Y. H., "Hybrid Analysis Method for Reliability-Based Design Optimization", Journal of Mechanical Design, Vol.125 (2003), pp.221-232.

[21] Youn, B. D. and Choi, K. K., "An Investigation of Nonlinearity of Reliability-Based Design Optimization Approaches", Journal of Mechanical Design, Vol.126 (2004), pp.403-411.

[22] Zhang, J. and Du, X., "A Second-Order Reliability Method with First-Order Efficiency", Journal of Mechanical Design, Vol.132, No. 10 (2010). 